\title{
Repetition and laterality effects on recognition memory for words and pictures*
}

\author{
JAMES F. JUOLA \\ University of Kansas, Lawrence, Kansas 66044
}

\begin{abstract}
Recognition memory for a list of words was tested by presenting a series of items with Ss instructed to make positive responses to targets (list items) and negative responses to distractors (nonlist items). The test items were either words or pictures, and they were presented tachistoscopically either to the left or right visual field. The results showed mean response latencies to be generally faster for stimuli presented to the right visual field. Response times were faster for target and distractor stimuli on their second test presentations than on initial tests, but this effect was much larger for targets. Repetitions were shown to decrease the amount of time necessary to execute the stimulus encoding and initial retrieval stages of recognition. This was also true, although to a lesser extent, if different stimulus forms (words or pictures) were used on the two tests. Subsequent recognition stages, including memory search and decision processes. were apparently independent of test stimulus form.
\end{abstract}

In recognition tests of memory. $\mathrm{S}$ is presented with a stimulus and he must categorize it as being either an "old" stimulus (i.e., one that has been presented before) or a "new" stimulus (i.e.. one that has not previously been presented). Any recognition paradigm is thus a special case of a discrimination task in which $S$ decides whether or not a given test stimulus is a member of a specific set of stimuli. For any recognition task there is, implicitly at least. a set of old, or target, stimuli and a set of new stimuli. or distractors. Correct recognition indicates the ability to identify from which set the test stimulus is drawn.

Experimental tests of recognition performance have usually begun with the presentation of the target set for study. To test memory for the target items, $S$ is presented with a number of stimuli. some of them targets and some distractors, and he is to say "old" or make a positive response to test items that he judges to be targets and to say "new" or make a negative response to distractors. In a study of short-term recognition memory, Sternberg (1966) presented a new set of from one to six different target digits on each trial. The presentation of the target set was followed by a single test digit. and $S$ was to make a positive response if the test digit was included in the target set and a negative response otherwise. Under these conditions, errors were very infrequent and the relevant data were response latencies. The results showed mean latencies to be linearly related to the number of items in the target set. Further, the slopes of the functions relating latencies to target set size were equal for positive and negative responses (i.e.. about $38 \mathrm{msec}$ per target digit). These

*This paper is based on a dissertation submitted to Stanford University in partial fulfillment of the requirements for a doctoral degree in psychology. I appreciate the assistance and encouragement of $R$. C. Atkinson. E. E. Smith. and I. Biederman. the members of my dissertation comnittee. results have been replicated in many other studies using a variety of stimulus materials besides digits, including letters (Chase \& Calfee, 1969), words (Juola \& A tkinson, 1971; Smith. 1967), faces (Sternberg, 1969), and geometric forms (Briggs \& Blaha, 1969).

The Stemberg paradigm has been extended to the study of long-term memory by Juola. Fischler, Wood, and Atkinson (1971). They presented sets of 10.18, or 26 words for Ss to memorize before the test session. The procedure then was the same as that used by Sternberg (1966), with a single test word presented on each trial. The results obtained by Juola et al were somewhat similar to those observed in the shorteterm memory studies in that the overall error rate was less than $5 \%$ and response times were increasing, linear functions of the number of target words. However, the slopes of the functions were much less in the long-term experiment (averaging about $5.0 \mathrm{msec}$ per item vs about $38 \mathrm{msec}$ per item in the Sternberg, 1966, study).

The model used by Sternberg $(1966,1969)$ to account for the data from short-term recognition studies assumes that total response latency can be regarded as a sum of the times necessary to execute several successive and independent stages between test stimulus onset and response output. These stages consist of (a) encoding the test stimulus into some internal representation that is compatible with the representations of the target set items. (b) a serial search. or scan. in which the test item is compared with each target item in memory, (c) a decision to make a positive or negative response based on the outcome of the search process. and (d) response selection and execution.

Juola et al (1971) and Atkinson and Juola (1973a. b) have proposed an alternative recognition model that employs a similar analysis of response latencies into execution times for the component stages. Three stages are proposed: an encoding stage. a decision stage. and a response stage. The encoding stage includes analysis of 


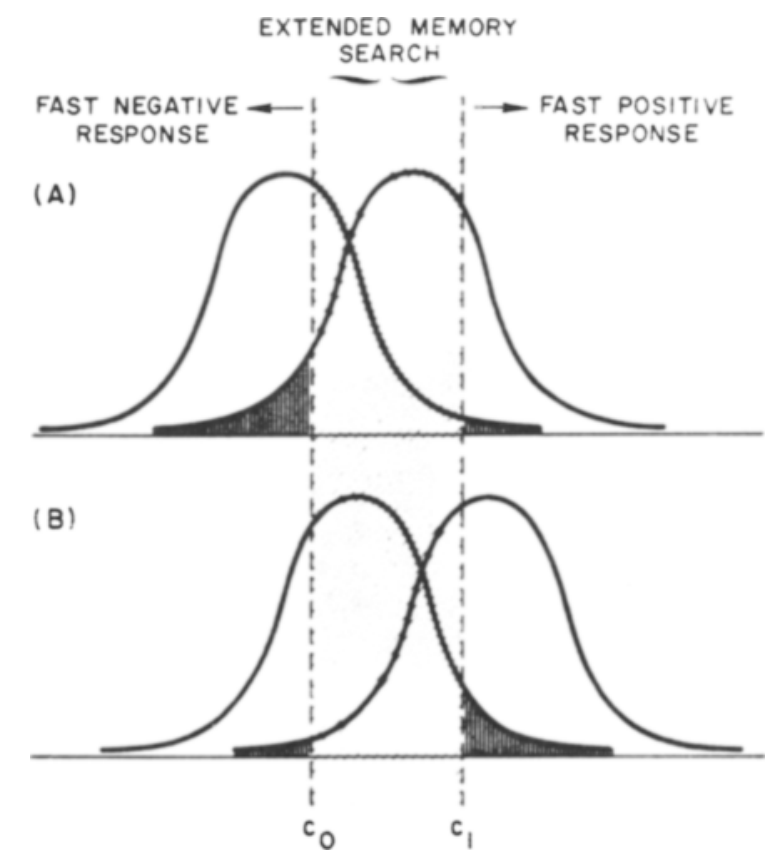

Fig. 1. Distributions of familiarity values for distractors (left) and targets (right) that have not been tested (Panel A) and for items that received a previous test presentation (Panel B).

the stimulus item and construction of an internal representation that allows direct access to the long-term memory location assigned to that item (e.g., Atkinson \& Juola, 1973b, have proposed that if the stimulus is a word, the first step in the recognition process is to access the appropriate node in the internal lexicon). Information associated with the test stimulus is then retrieved from long-term memory and is used by $S$ to arrive at an index of the item's strength or familiarity. The distributions of familiarity values for targets and distractors are presented in Fig. 1 (Panel A). The decision stage operates on the familiarity value output by the encoding stage. If this value is above a high criterion $\left(c_{1}\right)$ or below a low criterion $\left(c_{0}\right)$, S exits immediately to the response stage, emitting a positive or negative response, respectively. If the familiarity is of an intermediate value, $\mathrm{S}$ will be less sure of which response to choose and will perform an extended search of memory (perhaps retrieving the target set and scanning it for the presence or absence of the test item) before responding. Since, according to the model, the length of the target set affects only the time to execute the extended search, the slopes of the functions relating response latencies to target set size should depend on the relative familiarities of target and distractor items and the criteria that $S$ adopts for the decision process. Quantitative predictions derived from the model have been used to fit data from a variety of recognition experiments. including those with target sets stored in long-term memory or in short-term memory (Atkinson \& Juola. 1973a. b).
One set of predictions that readily derives from the model concerns the effects of repeated tests of specific target and distractor items on recognition performance. As shown in Fig. 1 (Panel B), repetitions are assumed to increase the familiarities of both targets and distractors. Thus, it would be expected that, when compared with the time to execute the decision stage on initial tests of target items, this stage should be executed more rapidly for repeated targets (since a smaller proportion of the items results in familiarities between $c_{0}$ and $c_{1}$ ), and it should be more accurate (since fewer target items will result in familiarities below ${ }^{\prime} c_{0}$ ). Similarly for distractors, repetitions should result in more false alarms and a slower mean time for the decision stage.

Besides these effects on the decision process, it is likely that repetitions also affect the encoding stage of recognition. There are several sources of evidence, some of them indirect, that indicate that the time taken for stimulus encoding and initial retrieval processes should be less for repeated stimuli than for stimuli presented for the first times in the context of the experiment. Studies of recognition thresholds for visual stimuli have shown that repetitions facilitate stimulus identification (Haber \& Hershenson, 1965; Dainoff \& Haber, 1967). Repetitions of stimuli in choice reaction time (CRT) tasks have resulted in faster response times (e.g. Bertelson, 1963), and this effect is obtained even if several presentations of different stimuli intervene between successive presentations of a given test stimulus (Remington, 1969). Several theoretical investigations of the repetition effect have indicated that the facilitative effect of repetitions is at least in part due to stimulus preparation and encoding biases that result in faster processing for repeated stimuli (Hinrichs \& Krainz, 1970: Keele, 1969: Theios \& Smith, 1972). Repetitions of stimuli in recognition tasks have also facilitated recognition performance over long-term retention intervals (Mahler. 1970). Taken together, these results indicate that the stimulus encoding stage should be executed more rapidly for repeated stimulus items.

In several recent studies of repetition effects on recognition performance (Atkinson \& Juola, 1973a; Hintzman, 1969; Juola et al, 1971; Okada, 1971), repetitions have resulted in faster mean response times and fewer errors for targets. Responses to repeated distractors have either been as fast as or slower than responses to initial presentations of distractor items, and the false alarm rate has generally increased with repetitions. In the previously cited studies of repetition effects on recognition, the effects of repetitions on encoding, retrieval, and decision processes have been confounded. This is the case because the studies have used a one-to-one mapping of stimuli to target and distractor items such that if any items in memory were to be tested more than once, the appropriate stimuli would also have to be repeated. Thus, repetitions of both target and distractor items should result in a similar. if not identical. facilitation of one or more processes associated with the encoding stage. However. 
as was argued earlier, repetitions should produce differential effects on the decision stage for targets and distractors. Repetition effects on the stages should be additive to produce faster mean response times for repeated target items. For repeated distractors. mean latencies could be either greater than or less than the times for responses to distractors on their first presentations, depending on the relative change in mean durations for the encoding and decision stages due to repetitions. Since earlier results have generally demonstrated that repetitions increase mean response latencies for distractors, it could be argued that the change in mean duration for the decision stage due to repetitions is opposite and also greater than the corresponding changes in encoding and retrieval times.

One of the main purposes of the present study was to provide direct evidence of a facilitation of the encoding stage of recognition due to repetitions of visual stimuli. Further. the study was designed to partial out the effects of repetitions on encoding and decision stages separately. This was done by using several different visual stimuli (i.e.. words or pictures presented tachistoscopically to the right or left visual hemifields) that corresponded to the same target or distractor items. Repetition effects could then be compared for the case in which the identical stimulus is used to test a given item in memory vs the case in which the stimulus associated with that item is different on successive tests. Although detailed predictions necessitate additional assumptions about such factors as the relative encoding times for words and pictures and laterality (i.e.. side of presentation) effects on encoding and memory processes. several hypotheses can be developed. First. mean encoding time is expected to be less for all repeated items when compared with the time necessary to encode items on their initial presentations. Any or all of the processes included in the encoding stage (i.e.. registration, identification, memory access, and information retrieval) could be executed faster in the case in which the identical stimulus is repeated. However, even if the form of the stimulus differs on the second test of any item. it is conceivable that some of the processes (e.g., accessing the appropriate memory location) could be executed more rapidly due to the previous exposure of an item with the same name and memorial referent. Second, the familiarity of any item is expected to depend only on whether it is a target or a distractor and whether or not it has previously been tested. This expectation is based on the strong assumption that the output of the encoding stage of the model is a familiarity value that is a function of the information about the test stimulus retrieved from long-term memory. Thus. the familiarity of any item should not depend on the form of the stimulus at time of test (as long as it is correctly identified). and it also might not depend on the form of the stimulus used in earlier tests of that item.

\section{METHOD}

\section{Subjects}

The Ss were 40 female Stanford University undergraduates. All Ss were right-handed and had normal or corrected-to-normal vision. Each was paid a total of $\$ 6$ for participation in three experimental sessions.

\section{Materials}

All stimuli were selected from a pool of 96 different items. The words were common one- or two-syllable nouns, three to eight letters in length. The pictures were simple outline drawings of the objects named by the nouns. The items were selected so that the words were all roughly equivalent in frequency and their corresponding pictures were easily and uniquely identifiable.

The stimuli were placed on $5 \times 9$ in. white cards for presentation in an Iconix tachistoscope. The words were typed with an IBM Executive typewriter and varied from 0.3 in. to 0.9 in. in length. Xerox copies of the original drawings were affixed to the cards, and they varied in width from 0.35 in. to 1.5 in. Two forms of each stimulus were made: one with the item on the left side of the card and one with the item on the right. When placed into the tachistoscope, the stimulus appeared no closer than 0.6 in. (1.1 deg of visual angle from S's viewpoint) to a central. dark fixation point. This separation insured that when the stimulus was exposed, the visual information would be registered solely in the contralateral cerebral hemisphere (Sperry, Gazzaniga. \& Bogen. 1969). In all future reference to the stimuli. the terms "left" and "right" will refer to the hemisphere that received the initial information input, not to the side of the visual field in which the stimulus appeared. Thus. there were four different stimuli for each of the 96 items: a word presented to the left hemisphere (WL), a word presented to the right hemisphere (WR). a picture presented to the left hemisphere (PL). and a picture presented to the right hemisphere (PR).

\section{Design}

The 96 items were randomly divided into two sets of 48 items each. The first set was used to generate target lists for the first $20 \mathrm{Ss}$, and the remaining 48 items were used as distractors. For the second group of $20 \mathrm{Ss}$, the target and distractor sets were reversed so that over the experiment as a whole, every item was used equally of ten as a target and as a distractor. Both target sets were randomly ordered into two different lists. so that four different target lists were used for four groups of 10 Ss each.

The test session consisted of 192 trials during which each item was tested twice. The order of presentation of items was randomly determined, with the constraint that no more than six target or distractor items could occur in succession. Six different test sequences were constructed. three for each target set. The test sequences were randomly assigned to $S$ s within each group. The first eight trials of every test sequence consisted of the four different types of test stimuli (WL. WR. PL. PR) presented twice each. once using a target item and once using a distractor. Trials 9-184 consisted of tests of target and distractor items that were presented for the first or second times distributed uniformly throughout the sequence. Trials $185-192$ consisted of the four different types of test stimuli presented once each with a repeated target item and a repeated distractor.

With four different types of stimuli available for each item. 16 different possible combinations of these stimuli existed for two tests of any item. Each combination was used equally often (three times) for targets and distractors. and they were distributed uniformly throughout the test sequences. Between 7 and 28 tests of other items occurred between successive tests of the same taroet or dividitor items. 


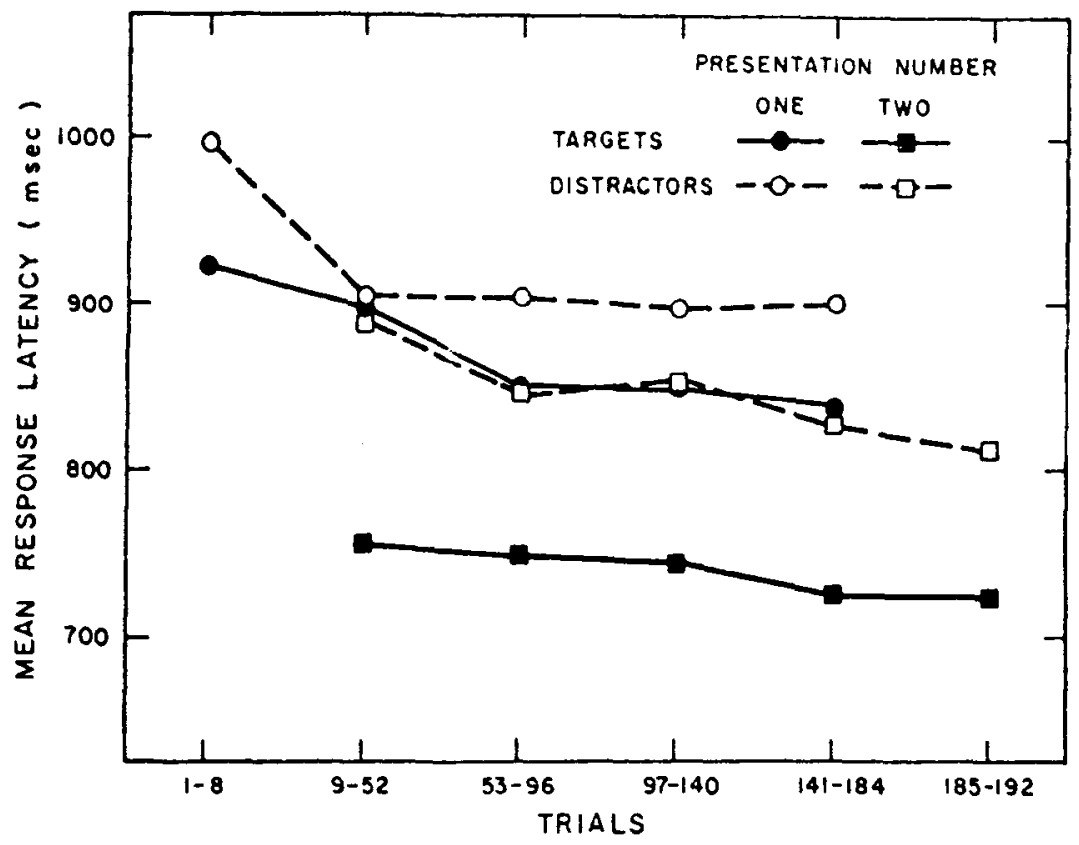

Fig. 2. Mean response latencies for target and distractor items on their first and second test presentations as functions of successive trial blocks.

Procedure

All Ss participated in three consecutive daily sessions. On Day 1. S was given a target list of 48 words to read through once. It was explained that the words in the list were to be memorized in the order that they appeared on the list and that this memorization was to be done sometime before the scheduled session on Day 2. The $S$ was then shown the target pictures and was asked to name each one as $E$ pointed to it. Almost all Ss correctly identified each of the pictures; any errors in identification were immediately corrected by $\mathrm{E}$.

The $\mathrm{S}$ was then seated in front of the tachistoscope, and the exact nature of the test procedure was explained. A series of 20 practice trials on a modified Sternberg task was run at the end of Day 1. For these trials, $\mathrm{S}$ was told to make a positive response if a digit from 0 to 4 was shown and to make a negative response if a digit from 5 to 9 was presented. All 10 digits were presented twice in a random order: once to the left and once to the right. The procedure for the practice trials was identical to that for the actual test sequence. Each trial started with $\mathrm{E}$ placing a test stimulus card into the tachistoscope and giving a ready signal to $\mathrm{S}$. The $\mathrm{S}$ then was to look directly at the central fixation point and press a start button operated by the left hand. The tachistoscope was programmed to maintain the preexposure field and fixation point for $200 \mathrm{msec}$ after $S$ pushed the start button. Then the stimulus card was exposed for $200 \mathrm{msec}$ followed by a dark field for $400 \mathrm{msec}$. The dark field was immediately followed by the onset of the preexposure field, which remained on until the next test. The $S$ made a positive or negative response by depressing one of two telegraph keys with the right forefinger. The two response keys were separated by a central home key on which $\mathrm{S}$ rested her finger between trials. All three keys were arranged along an arc, about $3 \mathrm{~cm}$ from one another, and the assignment of positive and negative responses to left and right keys was counterbalanced across $S$ s.

Day 2 began with a written serial recall test for the target words. All Ss correctly recalled their lists in one or two trials. The procedure for the test sequence was then reviewed, and 20 practice trials were run using the digits as test stimuli. This was followed by a brief rest period during which $S$ was reminded to respond as rapidly as possible while being careful to avoid making errors. The test sequence of 192 trials was then begun. No feedback was given for correct responses. but $S$ was informed of all errors. The $\mathrm{S}$ was allowed two rest periods at irregular intervals during the sequence. The actual test sessions lasted about $45 \mathrm{~min}$.

The Ss were scheduled to return on Day 3 for another test. the nature of which was unspecified. The third session consisted of a multiple-choice recognition test for information about the distractor items that had been incidentally learned during the previous day's test session. This delayed recognition test contained a list of 48 items. Each item consisted of three words typed in a row, one of which had been presented as a distractor on two trials in the test session. The three words were followed by the letters "W," "P." and "B." The S was instructed to circle the word that she remembered seeing during the previous day's session and then circle the appropriate letter to indicate whether she remembered it as being tested as a word (W), as a picture (P). or as both a word and a picture (B). The $S$ was told to respond to each item, even if she had to guess. The incorrect words in the multiple-choice test were matched as closely as possible with the experimental words in frequency, length, and concreteness. By rearranging the items, four completely different test sheets were constructed and were used by $10 \mathrm{Ss}$ each.

\section{RESULTS}

Figure 2 presents mean latencies for correct responses to target and distractor stimuli on their first and second test presentations. The data are presented for consecutive blocks of trials. The first block consisted of Trials $1-8$ (which included only first tests of target and distractor items). the next four blocks contained 44 trials each (in which both first and second tests were included), and the last block consisted of the final 8 trials (including only second test presentations). There appears to be some evidence for a general practice effect; that is, for all types of trials, response times were somewhat slower in the initial trial blocks than later in the session.

To eliminate the confounding of practice with repetition effects. only the data from Trials 9-184 were used for subsequent analyses. For this block of trials. the 
Table 1

Mean Response Latencies and Proportions of Errors (in Parentheses) for the Four Types of Target and Distractor Stimuli on Their First and Second Test Presentations

\begin{tabular}{|c|c|c|c|c|c|c|c|c|}
\hline \multirow{3}{*}{$\begin{array}{c}\text { Presen- } \\
\text { tation } \\
\text { Number }\end{array}$} & \multicolumn{4}{|c|}{ Targets } & \multicolumn{4}{|c|}{ Distractors } \\
\hline & \multicolumn{2}{|c|}{ Words } & \multicolumn{2}{|c|}{ Pictures } & \multicolumn{2}{|c|}{ Words } & \multicolumn{2}{|c|}{ Pictures } \\
\hline & Left & Right & Left & Right & Left & Right & Left & Right \\
\hline 1 & $\begin{array}{c}852 \\
(.164)\end{array}$ & $\begin{array}{c}876 \\
(.122)\end{array}$ & $\begin{array}{c}840 \\
(.150)\end{array}$ & $\begin{array}{c}863 \\
(.129)\end{array}$ & $\begin{array}{c}836 \\
(.017)\end{array}$ & $\begin{array}{c}872 \\
(.024)\end{array}$ & $\begin{array}{c}981 \\
(.065)\end{array}$ & $\begin{array}{c}956 \\
(.072)\end{array}$ \\
\hline 2 & $\begin{array}{c}734 \\
(.030)\end{array}$ & $\begin{array}{c}763 \\
(.017)\end{array}$ & $\begin{array}{c}728 \\
(.024)\end{array}$ & $\begin{array}{c}755 \\
(.031)\end{array}$ & $\begin{array}{c}836 \\
(.047)\end{array}$ & $\begin{array}{c}844 \\
(.055)\end{array}$ & $\begin{array}{c}876 \\
(.067)\end{array}$ & $\begin{array}{c}872 \\
(.060)\end{array}$ \\
\hline
\end{tabular}

overall error rate was $6.55 \%$. Table 1 presents mean latencies for correct responses and error proportions for target and distractor items on their first and second test presentations. The data for each type of stimulus (i.e., WL, WR, PL, and PR) are shown separately. A separate 2 by 2 analysis of variance was used to test for laterality and repetition effects on response latencies and error rates for target words, target pictures, distractor words, and distractor pictures. The results of these analyses showed mean response latency to be significantly shorter for repeated target items than for targets on their first presentations. This was true both for target words $[F(1.39)=165.34, p<.001]$ and for target pictures $[\mathrm{F}(1,39)=76.78, \mathrm{p}<.001]$. For distractor items, repetitions significantly decreased mean response times for pictures $[F(1.39)=22.60, p<.001]$ : however. the effect for distractor words was not significant $[F(1,39)=$ 2.47. $\mathrm{p}>.05]$

The hemisphere to which the stimuli were presented had a significant effect on positive response latency, with mean response times being shorter if the target items were presented to the left hemisphere [for target words. $F(1,39)=6.30, p<.05$, and for target pictures, $F(1,39)=7.46, p<.01]$. The results followed different patterns for distractor stimuli. with response times for words being faster if they were presented to the left and faster for pictures if they were presented to the right. However. neither main effect proved to be significant [for distractor words. $F(1.39)=2.93 . p>.05$, and for distractor pictures. $\mathrm{F}<1.0]$. The interactions between presentation number and input hemisphere were not significant for targets or for distractor pictures $(F<1.0$ in all three cases). but the interaction for distractor words was marginally significant $[F(1.39)=4.10$. $p \approx .05]$.

Repetitions resulted in a much lower error rate for target stimuli $[F(1.39)=48.41 . p<.001$ for words. and $\mathrm{F}(1.39)=9.30 . \mathrm{p}<.01$ for pictures]. For distractors. the error rate was higher for words presented for the second times than for those on their first tests $[F(1.39)$ $=9.30 . p<.01]$. but there was no change in the error rate for repeated pictures $(F<1.0)$. The error rate for target words was somewhat greater for stimuli that were presented to the left $[F(1.39)=4.35 . p<.05]$. There were no other significant laterality effects on the error rates, and none of the interactions between repetitions and input hemisphere were significant.

Table 2 presents mean response latencies and error proportions for second test presentations of target and distractor stimuli conditionalized on whether or not the first test was of the same stimulus form (i.e., word or picture) and whether or not it was presented to the same hemisphere. Separate 2 by 2 analyses of variance were run on the latency and error data for targets and distractors. For the latency data, the main effect of stimulus form was significant for targets $[F(1.39)=$ $84.50, p<.001]$ and for distractors $[F(1,39)=24.19$. $p<.001]$, but there were no significant effects due to the hemisphere to which stimuli were presented. The interaction between stimulus form and presentation hemisphere was also nonsignificant in both cases. and none of the main effects or their interactions approached significance for the error data.

In Table 3. mean response latencies and error proportions for second test presentations are shown for the two stimulus forms conditionalized on the stimulus forms used on the first tests of the items. Separate $2 \mathrm{by}$ 2 analyses of variance were run on the latency and error data for targets and distractors. Response times to second tests of target stimuli were significantly faster if the first presentations were pictures than if they were words $[F(1,39)=21.86, p<.001]$. The forms of the stimuli used for the second test presentation had no effect on response latency. but the interaction between forms used on the first and second presentations was highly significant $[F(1.39)=79.50 . p<.001]$. For

Table 2

Mean Response Latencies and Error Proportions (in Parentheses) for Second Test Presentations of Target and Distractor Items Conditionalized on Whether or Not the Stimulus From and Hemisphere of Input Were the Same on the First Test of the Item

\begin{tabular}{|c|c|c|c|c|}
\hline & \multicolumn{2}{|c|}{ Targets } & \multicolumn{2}{|c|}{ Distractors } \\
\hline & $\begin{array}{c}\text { Same } \\
\text { Stimulus }\end{array}$ & $\begin{array}{l}\text { Different } \\
\text { Stimulus }\end{array}$ & $\begin{array}{c}\text { Same } \\
\text { Stimulus }\end{array}$ & $\begin{array}{l}\text { Different } \\
\text { Stimulus }\end{array}$ \\
\hline $\begin{array}{l}\text { Same } \\
\text { Hemisphere }\end{array}$ & $\begin{array}{c}710 \\
(.028)\end{array}$ & $\begin{array}{c}780 \\
(.036)\end{array}$ & $\begin{array}{c}836 \\
1.060)\end{array}$ & $\begin{array}{c}874 \\
1.0521\end{array}$ \\
\hline $\begin{array}{l}\text { Different } \\
\text { Hemisphere }\end{array}$ & $\begin{array}{c}713 \\
0 .(115)\end{array}$ & $\begin{array}{l}776 \\
1.0221\end{array}$ & $\begin{array}{c}823 \\
1.6381\end{array}$ & $\begin{array}{c}903 \\
1.06+1\end{array}$ \\
\hline
\end{tabular}


Table 3

Mean Response Latencies and Error Proportions (in Parentheses) for Second Test Presentations of Target and Distractor Items Conditionalized on the Forms of the Stimuli Tested

\begin{tabular}{cccccc}
\hline & \multicolumn{2}{c}{ Targets } & & \multicolumn{2}{c}{ Distractors } \\
\cline { 3 - 3 } \cline { 5 - 6 } Test Stimulus & Word & Picture & & Word & Picture \\
\hline Second & & & & \\
Test Stimulus & & & 827 & 854 \\
Word & 729 & 766 & & & \\
& $(.023)$ & $(.024)$ & & $(.067)$ & $(.037)$ \\
Picture & 792 & 693 & 923 & 834 \\
& $(.034)$ & $(.021)$ & $(.076)$ & $(.053)$ \\
\hline
\end{tabular}

distractors, response times on second tests were faster if pictures were used in the first tests $[F(1,39)=5.00$, $p<.05]$ and if words were used in the second tests $[F(1.39)=11.06, p<.01]$. The interaction was also significant $[F(1,39)=23.65, p<.001]$. For both targets and distractors, the significant interactions show that the latency of a response to any stimulus was faster if the same stimulus form had been used for the earlier test of that item than if the other form had been presented.

Neither of the main effects nor the interaction was significant for the error data for targets shown in Table 3. For distractors, significantly more errors were made on second test presentations for those items that had been presented as words on the first tests $[F(1,39)=$ 6.03. $p<.05]$. The other main effect and the interaction were not significant.

A summary of the response latency data is presented in Fig. 3. Mean latencies are shown for target items (left panel) and for distractor items (right panel) on their first and second test presentations. The straight lines connect data points for items that were tested either with the same or different stimulus forms in each test. In all cases, mean response times for targets on their second tests were significantly faster than the times for responses on their initial tests. Even the smallest difference (i.e.. that between mean latencies for responses to target pictures on their initial tests and latencies for responses to target pictures on second presentations if the items had been presented as words on the first tests) was obtained for 32 of the 40 Ss (27 of $40 \mathrm{Ss}$ must show a trend for it to be significant at the .05 level by a sign test). For distractors, responses to repeated items were faster than were responses to initial tests for both pictures and words if the items had been presented as pictures on their first tests (this trend was shown by 32 of $40 \mathrm{Ss}$ for items presented as pictures on the first tests and as words on the second tests, and it was obtained for $38 \mathrm{Ss}$ for items presented as pictures on both tests). For distractor items presented as words on the first tests, responses to second presentations were not significantly faster if words were tested, but responses were significantly slower if pictures were presented on the second tests (this trend was obtained for $30 \mathrm{Ss}$ ). A final interesting comparison is between response times for distractor pictures on initial tests and for distractor pictures on their second tests if the items had been tested as words on their first presentations. Although only 26 of 40 Ss were faster in the latter case. the difference was significant $[t(39)=1.97, p<.05]$.

In the delayed recognition test, Ss were to pick out. from among three alternatives in each case, the items that had been presented twice as distractors during the previous day's session. These items were correctly chosen in $94.2 \%$ of the cases. The distribution of errors over stimulus forms is presented in Table 4. A Wilcoxon signed-ranks test showed that significantly more errors

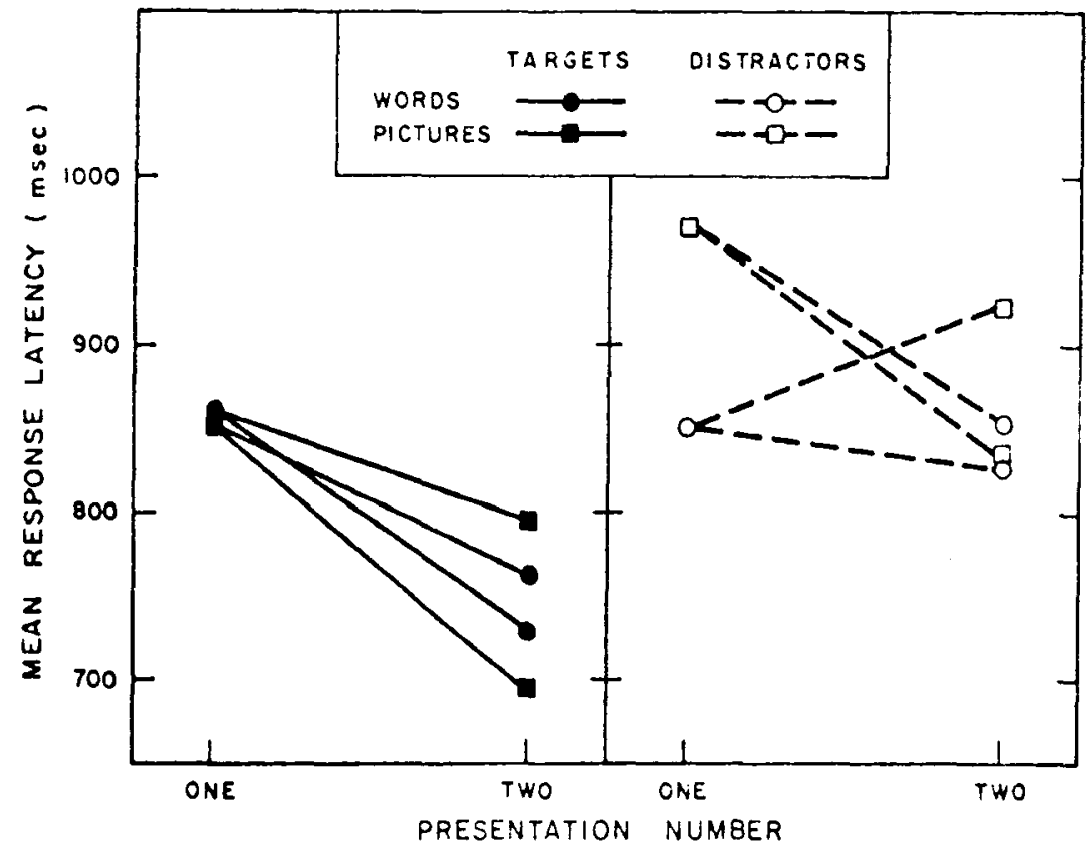

Fig. 3. Mean response latencies for target and distractor items as functions of the forms of the stimuli used for the first and second test presentations. 
were made for items that were presented twice as words than for those presented twice as pictures. Table 5 presents errors for items that were presented twice to the left hemisphere, twice to the right hemisphere, or once to each hemisphere. A Wilcoxon test showed that more errors were made for items presented twice to the right than for those presented twice to the left, although this effect was only marginally significant $(p=.05)$.

The Ss were also asked to indicate the form of the stimulus (word. picture, or both) that they thought had been used to test the distractor items. The relative proportions chosen for each form conditionalized on the actual form presented are shown in Table 6. The responses for those items that were correctly recognized and for those items that Ss did not recognize are presented separately.

\section{DISCUSSION}

Repeated presentations of target and distractor items in a recognition task have a large and differential effect on positive and negative responses. For target items, the results replicated earlier findings (Atkinson \& Juola, 1973a; Fischler \& Juola. 1971; Hintzman, 1969: Okada. 1971 ) in that mean response latencies were shorter and error rates were lower on second test presentations than on initial tests. The repetition effects for distractor items differed from those obtained for targets as well as from the results of earlier studies. Although Juola et al (1971) report a nonsignificant increase in negative response latencies due to repeated test presentations of distractor words. later studies (Atkinson \& Juola. 1973a: Fischler \& Juola. 1971) have demonstrated reliable increases in both mean latencies and error rates for repeated distractors. All of these previous experiments have used words as stimuli. In the present study, there was no significant change in response latency for distractor words as a function of repetitions: however, the false alarm rate was greater for distractor words on their second tests than on initial presentations. For repeated distractor pictures, mean latency was shorter but the error rate was about the same as on first test presentations.

In terms of the recognition model discussed earlier. repetitions of test stimuli result in faster stimulus encoding and initial retrieval times as well as higher familiarity values for both target and distractor items. The relative familiarities of targets and distractors on their first and second tests can be estimated. in most cases. directly from the error rates. The results were generally in accord with this formulization. The lower error rates for repeated targets indicate that repetitions increased their familiarities. resulting as well in a shorter mean time to execute the decision stage (as diagrammed in Fig. 1). The reduction in decision time. combined with a shorter duration for the encoding stage. resulted in a much shorter overall latency for repeated targets. The fact that there were no differences in the results for
Table 4

Mean Proportions of Errors for Distractor Items that Had Been Presented Twice as Words, Twice as Pictures, or Once Each as a Word and as a Picture (Delayed Recognition Test)

\begin{tabular}{ccc}
\hline Words & Pictures & Both \\
\hline .083 & .046 & .051 \\
\hline
\end{tabular}

Table 5

Mean Proportions of Errors for Distractor Items that Had Been Presented Twice to the Left Hemisphere, Twice to the Right Hemisphere, or Once to Each Hemisphere (Delayed Recognition Test)

\begin{tabular}{ccc}
\hline Left & Right & Both \\
\hline .048 & .071 & .056 \\
\hline
\end{tabular}

Table 6

Relative Proportions on Items that Ss Indicated Had Occurred Twice as Words, Twice as Pictures, or Once Each as a Word and as a Picture for Items that Had Actually Been Presented as Words, Pictures, or Both (Delayed Recognition Test)

\begin{tabular}{lcccc}
\hline $\begin{array}{c}\text { S Indi- } \\
\text { cated that } \\
\text { Item Had } \\
\text { Occurred as }\end{array}$ & $\begin{array}{c}\text { Distractor Item Correctly } \\
\text { Recognized and Presented as }\end{array}$ & $\begin{array}{r}\text { Distractor } \\
\text { Item Not } \\
\text { Correctly } \\
\text { Recognized }\end{array}$ \\
\hline Words & Words & Pictures & Both & Recogict \\
Pictures & .63 & .03 & .17 & .42 \\
Both & .13 & .71 & .27 & .42 \\
\hline
\end{tabular}

target words and target pictures indicates that the length of time necessary to execute the encoding stage as well as the familiarities of the test items (and thereby the amount of time to execute the decision stage) were equivalent for the two stimulus forms.

The differences between the results for distractor words and distractor pictures can also be interpreted in terms of the model. Repetitions of distractor words resulted in a higher false alarm rate. indicating a higher familiarity value and thus an expected increase in the mean decision time. Since mean response times were approximately the same for first and second tests of distractor words. it could be argued that the increase in decision time due to repetitions was about equal to the decrease in encoding time that also resulted from repetitions. Thus, in the present study. the two effects might have cancelled one another. whereas in other experiments (e.g.. Fischler \& Juola. 1971). the change in decision time was the overriding factor. resulting in an increase in total response time for repeated distractors. This result was not unexpected in that. in the present study, stimuli were presented peripherally for $200 \mathrm{msec}$. whereas in the Fischler and Juola experiment. the stimuli were exposed foveally for $500 \mathrm{msec}$. Thus. the encoding stage would be expected to require a greater execution time in the present study. and it might also show a greater absolute effect of stimulus repetition.

The error rate for distractor pictures was greater than that for words, but there was no shange in the number 
of false alarms from the first test presentation to the second. It is possible that these errors could indicate a higher familiarity for pictures, but it is more likely that (as for targets) distractor words and pictures were about equally familiar. The higher error rate for distractor pictures. especially on initial tests, might well have been due entirely to a greater probability of incorrectly identifying the test stimulus. Unlike the target pictures, which had been presented for study on the day before the test session. the distractor pictures were shown only as test stimuli. and Ss had never seen them before. Therefore, the length of time necessary to execute the encoding stage should be greatest for distractor pictures on their first presentations. If the exposure duration was insufficient for identification, $S$ either did not respond or guessed on the basis of incomplete information (and was more likely to be incorrect). Both the probability of incorrectly identifying the distractor picture and the time necessary to encode it should be less on the second test presentation. Thus, the obtained pattern of results is expected as long as it is assumed that the encoding time for distractor pictures is not only greater than that for words, but that it is also more affected by repetitions.

The results indicated that the cerebral hemisphere to which the stimuli were presented also had an effect on recognition performance. In the present task, there are at least two reasons why performance should be superior for stimuli presented to the left hemisphere than for stimuli shown to the right: (1) The task involves decisions about verbal materials, i.e., whether or not a given test item is a member of a memorized list of words. It is likely that this task, since it requires the retrieval of verbal information from long-term memory, can be best performed if the test stimulus is input directly to the language-dominant hemisphere. This would be the left hemisphere for the vast majority of Ss in the present study (all Ss were right-handed). Thus, stimuli presented to the left hemisphere could be processed immediately, whereas information presented to the right hemisphere would have to be transferred to the left before processing could continue. This transfer should add about $10-40 \mathrm{msec}$ to the total response time (Filbey \& Gazzaniga, 1969; Moscovitch \& Catlin, 1970). (2) Studies of perceptual recognition have generally shown that for the classes of stimuli used in the present study (i.e.. words and simple outline drawings of common objects), identification is superior for information presented to the left hemisphere (Bryden \& Rainey, 1963; Wyke \& Ettlinger, 1961).

The results (as shown in Table 1) generally agreed with the predicted advantage for the left hemisphere. Response latencies were shorter if target words, target pictures, and distractor words were presented to the left hemisphere, although the latter effect did not achieve statistical significance. For distractor pictures, response latencies actually were shorter if the stimuli were presented to the right hemisphere, but this effect was also not significant. The opposite trend for distractor pictures is also probably related to the fact that they were new to Ss and therefore they were more difficult to identify than were the target pictures. In cases where there has been no difference between right and left presentation on recognition or where the right hemisphere has shown an advantage, the stimuli have been nonsense forms or other visual materials without an immediately available verbal label (Terrace, 1959: White, 1969). A similar phenomenon might account for the results for distractors, especially picture stimuli, in the present study.

Although the hemisphere to which stimuli were presented affected responses at the time of test, responses to items on their second tests were not affected by the input hemisphere for the first tests of the items. Table 2 shows the results for second tests conditionalized on the types of stimuli used for the first and second tests of target and distractor items. For both targets and distractors, responses were faster if the stimuli used in the second tests were of the same form (words or pictures) as those for initial tests. However, there was no significant effect due to whether or not the stimulus had been previously presented to the same hemisphere, and none of the differences in the error rates were significant.

An interpretation of the results for repeated items can now be made in terms of the recognition model. It is assumed that the relative familiarity of any test item can be estimated from the error rate for that item, with the exceptions being those stimuli (i.e., distractor pictures the first times they are presented) that result in higher error rates due to a relatively greater probability of being incorrectly identified. Repeated tests increase the familiarity values for all items and result in an increased error rate for distractors and a decreased error rate for targets. An important result of the present study is that familiarity does not appear to be affected by the form of the stimulus used to test recognition for specific target or distractor items. Error rates were the same for all repeated target stimuli and for all repeated distractor stimuli (with the one exception being items that were tested first as words then as pictures) regardless of whether or not the same form of the stimulus was used on both tests.

That responses were faster for both target and distractor items if the stimuli were of the same form on both tests indicates that either or both of the component processes of the encoding stage (defined as stimulus encoding plus the initial retrieval process) were executed more rapidly for these items. The output of the encoding stage, according to the model, is a subjective familiarity value that is not affected by the stimulus form used to test the item on that trial or on any previous presentations. A strong prediction that can be derived from this conceptualization is that the difference between mean response latencies for those items that were tested with the same stimulus form on both presentations and those that were tested with 
different stimuli should be the same for targets and distractors. This prediction is based on two assumptions: (1) The savings in the encoding time due to repetitions of identical stimuli is the same for targets and distractors. (This assumption might prove to be unwarranted, but there is no evidence in the present data on which to base alternative arguments.) (2) As stated earlier, familiarity is based on the information recovered from long-term memory by the initial retrieval process. Whereas this process is assumed to execute more rapidly for repeated items, it is not necessary to assume that either its execution time or the resultant familiarity value is influenced by the form of the stimulus. If familiarity were affected by stimulus form, it would be natural to assume that an item's familiarity would be greater if the same form were used on successive tests than if different forms of the same item were presented. In this case, the time necessary to execute the decision stage would be less for repeated targets if the stimulus form were the same, and this time would be greater for repeated distractors if the form were the same than if it were different on the two tests.

The results showed that the latency data agreed with the error data in indicating that item familiarity does not depend on the form of the stimulus that represents the item. Refrring to Table 3, it can be observed that mean response htency for target words on their second tests was $37 \mathrm{msec}$ less if the first test stimuli also were words than if they were pictures. This difference was $27 \mathrm{msec}$ for distractors. Similarly. mean latency for responses to target pictures was $99 \mathrm{msec}$ less if the first stimuli used to test these items were pictures than if they were words. For distractors. this difference was $89 \mathrm{msec}$. Neither $10-\mathrm{msec}$ difference was significant. The faster times for repeated items if the stimuli were also repeated can be accounted for by assuming that the stimulus encoding process alone is influenced by stimulus form and that it is executed more rapidly for repeated stimuli than for alternate stimulus forms of previously tested items. It was argued earlier that repetitions facilitate encoding more for pictures than for words. The data shown in Table 3 support this contention, and it is further indicated that the repetition effect on encoding times might be equivalent for target and distractor stimuli.

The data presented in Fig. 5 allow for comparisons between response latencies for the conditional response times shown in Table 3 and the times for responses to initial presentations of target and distractor stimuli. All four of the data points for repeated targets are significantly below those for initial presentations of target items. These results are not surprising in that the initial retrieval and decision processes are expected to be executed more rapidly for repeated targets. In addition, the stimulus encoding process is faster at least for those repeated items for which the stimulus form was also repeated. Further inferences about the relative effects of repetitions on the processing stages are difficult to make because of the confounding of the facilitative effects of repetitions on retrieval and decision times for positive responses.

For the data for negative trials, however, the effects of repetitions are opposite for the decision stage and for the stimulus encoding and initial retrieval components of the encoding stage. Because of the relatively large amount of time necessary to encode distractor pictures on their first presentations, responses to these stimuli are significantly slower than to distractor words or to pictures on their second presentations. Some conclusions can be drawn about the relative effects of repetitions on the separate processing stages by comparing latencies for responses to distractor words and pictures on their first presentations with latencies of responses to distractor items on their second tests if the stimulus forms were different on the two tests. The question to be answered is: Do exposures of one form of a stimulus facilitate the encoding process for a subsequent presentation of the item with a different stimulus form? Studies of perceptual recognition for pictures (Lawrence \& Coles, 1954; Pachella, 1970) have consistently shown that the presentation of one or more name alternatives before the tachistoscopic exposure of a test pictures does not facilitate its recognition. In the present study. response times to distractor words on their initial tests were approximately the same as response times to distractor words that had previously been tested as pictures. This result indicates that the increase in decision time due to the previous test of the item is about equal to the decrease in stimulus encoding time (if any) and in the initial retrieval time. Response times to distractor pictures on their initial tests, however, were significantly slower than were response latencies to distractor pictures that had previously been tested as words. Since it was earlier argued that the initial retrieval process and the decision stage are independent of stimulus form, this effect can only be attributed to a differential facilitation of the stimulus encoding process for words and pictures due to previous exposure of a different form of the same item. That is, the presentation of a distractor word results in a faster encoding time for the subsequent presentation of the associated picture than the encoding time for initially presented distractor pictures. Although it cannot be determined if there is a facilitative effect on the encoding time for distractor words due to previous presentation of the associated pictures, this effect (if any) is smaller than that for distractor pictures.

The delayed recognition data indicated that even after a 24 -h delay. Ss remembered the forms of the stimuli in about $50 \%$ of the cases (data from Table 6. corrected for guessing). Thus, the form of the stimulus presented at the time of test is stored in long-term memory; however. this information apparently is not used in determining the familiarity value or in making a response decision about the stimulus. The only information that is important in influencing recognition is contextual and recency information retrieved about the test stimulus. 
Thus, recognition performance is determined, chiefly, by only two factors: whether or not the test stimulus represents an item in the target set. and the length of time since the long-term memory location assigned to the test stimulus has last been accessed.

\section{REFERENCES}

Atkinson. R. C., \& Juola, J. F. Factors influencing speed and accuracy of word recognition. In S. Kornblum (Ed.), Attention and performance IV. New York: Academic Press, 1973 a.

Atkinson, R. C., \& Juola, J. F. Search and decision processes in recognition memory. In D. Krantz, R. C. Atkinson, R. D. Luce, and P. Suppes (Eds.), Contemporary developments in mathematical psychology. New York: Academic Press, 1973b.

Bertelson, P. S-R relationships and reaction times to new versus repeated signais in a serial task. Journal of Experimental Psychology, 1963, 65, 478-484.

Briggs, G. E., \& Blaha, J. Memory retrieval and central comparison times in information processing. Journal of Experimental Psychology, 1969, 79, 395-402.

Bryden, M. P., \& Rainey, C. A. Left-right differences in tachistoscopic recognition. Journal of Experimental Psychology, 1963, 66, 568-571.

Chase, W. G., \& Calfee, R. C. Modality and similarity effects in short-term recognition memory. Journal of Experimental Psychology, 1969, 81. 510-514.

Dainoff, M.. \& Haber, R. N. How much help do repeated presentations give to recognition processes? Perception \& Psychophysics, 1967, 2, 131-136.

Filbey. R. A., \& Gazzaniga, M. S. Splitting the normal brain with reaction time. Psychonomic Science, 1969, 17, 335-336.

Fischler. I., \& Juola, J. F. Effects of repeated tests on recognition time for information in long-term memory. Journal of Experimental Psychology, 1971, 91, 54-58.

Haber, R. N., \& Hershenson, M. Effects of repeated brief exposures on the growth of a percept. Journal of Experimental Psychology, 1965, 69, 40-46.

Hinrichs, J. V., \& Krainz, P. L. Expectancy in choice reaction time: Anticipation of stimulus or response? Journal of Experimental Psychology, 1970, 85, 330-334.

Hintzman, D. Recognition time: Effects or recency, frequency, and the spacing of repetitions. Journal of Experimental Psychology, 1969, 79, 192-194.

Juola, J. F., \& Atkinson, R. C. Memory scanning for words versus categories. Journal of Verbal Learning \& Verbal Behavior, 1971, 10, 522-527.

Juola, J. F., Fischler. I., Wood, C. T., \& Atkinson, R. C. Recognition time for information stored in long-term memory. Perception \& Psychophysics, 1971, 10, 8-14.

Keele, S. W. The repetition effect: A memory dependent process. Journal of Experimental Psychology, 1969, 80, 243-248.

Lawrence, D. H., \& Coles, G. R. Accuracy of recognition with alternatives before and after the stimulus. Journal of Experimental Psychology, 1954, 47, 208-214.

Mahler, W. A. Effects of information retrieval on human memory. Unpublished doctoral dissertation, Stanford University, 1970.

Moscovitch, M., \& Catlin, J. Interhemispheric transmission of information: Measurement in normal man. Psychonomic Science, 1970, 18, 211-213.

Okada, R. Decision latencies in short-term recognition memory. Journal of Experimental Psychology, 1971, 90, 27-32.

Pachella, R. G. The nature of the effect of set on tachistoscopic recognition. Technical Memorandum 21-70, 1970, Human Engineering Laboratories, Aberdeen Proving Ground, Maryland.

Remington, R. J. Analysis of sequential effects in choice reaction times. Journal of Experimental Psychology, 1969, 82, 250-257.

Smith. E. E. Effects of familiarity on stimulus recognition and categorization. Journal of Experimental Psychology, 1967, 74, 324-332.

Sperry, R. W., Gazzaniga, M. S., \& Bogen, J. E. Interhemispheric relationships: The neocortical commissures: Syndromes of hemisphere disconnection. In P. J. Vinken and G. W. Bruyn (Eds.), Handbook of clinical neurology. Vol 4. Amsterdam: North-Holland, 1969.

Stemberg. S. High-speed scanning in human memory. Science. $1966,153,652-654$

Sternberg, S. Memory scanning: Mental processes revealed by reaction-time experiments. American Scientist. 1969, 57, 421-457.

Terrace, H. S. The effects of retinal locus and attention on the perception of words. Journal of Experimental Psychology, $1959,58,382-385$.

Theios, J., \& Smith, P. G. Can a two-state model account for two-choice reaction-time data? Psychological Review, 1972. 79, $172-177$.

White, M. J. Laterality differences in perception: A review. Psychological Bulletin, 1969, 72, 387-405.

Wyke, M., \& Ettlinger, G. Ffficiency of recognition in left and right visual fields. Archives of Neurology, 1961, 5. 659-665.

(Received for publication December 28, 1972; accepted January 7,1973 .) 